\title{
Multicenter Phase II Study of Oxaliplatin, Irinotecan, and S-1 as First-Line Treatment for Patients with Recurrent or Metastatic Biliary Tract Cancer
}

\author{
Changhoon Yoo, MD 1 \\ Boram Han, MD, PhD² \\ Hyeong Su Kim, MD, PhD² \\ Kyu-pyo Kim, MD, PhD' \\ Deokhoon Kim, PhD'3,4 \\ Jae Ho Jeong, MD ${ }^{1}$ \\ Jae-Lyun Lee, MD, $\mathrm{PhD}{ }^{1}$ \\ Tae Won Kim, MD, PhD ${ }^{1}$ \\ Jung Han Kim, MD, PhD² \\ Dae Ro Choi, MD, PhD² \\ Hong II Ha, MD, PhD 5 \\ Jinwon Seo, MD, PhD ${ }^{6}$ \\ Heung-Moon Chang, MD, PhD' \\ Baek-Yeol Ryoo, MD, PhD ${ }^{1}$ \\ Dae Young Zang, MD, PhD²
}

*A list author's affiliations appears at the end of the paper.

Correspondence: Dae Young Zang, MD, PhD Division of Hematology-Oncology,

Department of Internal Medicine,

Hallym University Sacred Heart Hospital,

Hallym University College of Medicine,

22 Gwanpyeong-ro 170beon-gil, Dongan-gu,

Anyang 14068, Korea

Tel: 82-31-380-1500

Fax: 82-31-380-1528

E-mail: fhdzang@hallym.ac.kr

Co-correspondence: Baek-Yeol Ryoo, MD, PhD Department of Oncology, Asan Medical Center, University of Ulsan College of Medicine,

88 Olympic-ro 43-gil, Songpa-gu,

Seoul 05505, Korea

Tel: 82-2-3010-3211

Fax: 82-2-3010-6961

E-mail: ryooby@amc.seoul.kr

Received October 31, 2017

Accepted January 4, 2018

Published Online January 8, 2018

${ }^{*}$ Changhoon Yoo and Boram Han contributed equally to this work.

\section{Purpose}

Although gemcitabine plus cisplatin has been established as the standard first-line chemotherapy for patients with advanced biliary tract cancer (BTC), overall prognosis remains poor. We investigated the efficacy of a novel triplet combination of oxaliplatin, irinotecan, and S-1 (OIS) for advanced BTC.

\section{Materials and Methods}

Chemotherapy-naive patients with histologically documented unresectable or metastatic BTC were eligible for this multicenter, single-arm phase II study. Patients received $65 \mathrm{mg} / \mathrm{m}^{2}$ oxaliplatin (day 1), $135 \mathrm{mg} / \mathrm{m}^{2}$ irinotecan (day 1), and $40 \mathrm{mg} / \mathrm{m}^{2} \mathrm{~S}-1$ (twice a day, days 1-7) every 2 weeks. Primary endpoint was objective response rate. Targeted exome sequencing for biomarker analysis was performed using archival tissue.

\section{Results}

In total, 32 patients were enrolled between October 2015 and June 2016. Median age was 64 years (range, 40 to 76 years), with 24 (75\%) male patients; $97 \%$ patients had metastatic or recurrent disease. Response rate was $50 \%$, and median progression-free survival and overall survival (OS) were 6.8 months ( $95 \%$ confidence interval [Cl], 4.8 to 8.8 ) and 12.5 months (95\% Cl, 7.0 to 18.0), respectively. The most common grade 3-4 adverse events were neutropenia (32\%), diarrhea (6\%), and peripheral neuropathy (6\%). TP53 and KRAS mutations were the most frequent genomic alterations ( $42 \%$ and $32 \%$, respectively), and KRAS mutations showed a marginal relationship with worse OS $(p=0.07)$.

\section{Conclusion}

OIS combination chemotherapy was feasible and associated with favorable efficacy outcomes as a first-line treatment in patients with advanced BTC. Randomized studies are needed to compare OIS with gemcitabine plus cisplatin.

\author{
Key words \\ Biliary tract neoplasms, Cholangiocarcinoma, Chemotherapy, \\ Irinotecan, Oxaliplatin, S-1
}




\section{Introduction}

Biliary tract cancer (BTC) is a rare malignancy comprising a heterogeneous group of diseases including intrahepatic/ extrahepatic cholangiocarcinoma and gallbladder cancer. Up to 10,000 new BTC patients are diagnosed annually in the United States and Europe [1]. In Korea, BTC is one of leading causes of cancer-related deaths (sixth in males and seventh in females) [2]. Surgical resection is the only curative therapeutic modality for localized BTC; however, recurrence after curative resection is common, and most patients present with unresectable or metastatic disease at the time of diagnosis. These clinical characteristics in advanced BTC are associated with poor prognosis, with up to $10 \%$ 5-year overall survival (OS) rates [3].

After the success of the pivotal phase III ABC-02 study, gemcitabine plus cisplatin (GemCis) has been established as the standard first-line chemotherapy for patients with unresectable or metastatic BTC [4]. In that study, GemCis demonstrated significantly improved OS compared with gemcitabine alone (11.7 months vs. 8.1 months). The superiority of GemCis to gemcitabine alone was confirmed in a subsequent Japanese randomized phase II study (11.2 months vs. 7.7 months) [5]. Despite this improvement, the median survival remains $<1$ year for patients with advanced BTC, highlighting the large unmet need for improving the efficacy of systemic chemotherapy.

Investigation of combination therapy including three cytotoxic chemotherapeutic agents has revealed a significant survival benefit in multiple cancer types [6,7]. Particularly in pancreatic cancer, FOLFIRINOX, the combination of fluorouracil, irinotecan, and oxaliplatin, leads to significantly improved survival outcomes compared with gemcitabine monotherapy; this has been globally accepted as one of standard first-line regimens for patients with locally advanced or metastatic pancreatic cancer [7]. Considering these success, gemcitabine-free triplets including irinotecan, oxaliplatin, and fluorouracil might also be effective in treating advanced BTC. Furthermore, in a previous phase I study, biweekly triplet combination of oxaliplatin, irinotecan, and S-1 (oral fluoropyrimidine) (OIS) for multiple cancer types was associated with remarkable preliminary efficacy outcomes in advanced BTC, with four of nine BTC patients achieving partial response (PR) [8].

Based on these findings, we herein report the results of a single-arm, phase II study that aimed to evaluate the efficacy and safety of OIS in patients with unresectable or metastatic BTC.

\section{Materials and Methods}

\section{Eligibility}

Patients with histologically confirmed BTC were eligible if they were chemotherapy-naive and had inoperable locally advanced or metastatic disease. Other inclusion criteria included age of $\geq 19$ years; measurable lesion according to the Response Evaluation Criteria in Solid Tumors (RECIST) ver. 1.1 [9]; Eastern Cooperative Oncology Group performance status of $0-2$; adequate bone marrow, renal, and hepatic function; life expectancy of $\geq 3$ months; and written informed consent. Patients were excluded if they had received chemotherapy for BTC. However, previous adjuvant chemotherapy without platinum was allowed if the interval between the completion of adjuvant chemotherapy and enrollment in the study was $>6$ months.

\section{Treatment}

Patients received intravenous $65-\mathrm{mg} / \mathrm{m}^{2}$ oxaliplatin and $135-\mathrm{mg} / \mathrm{m}^{2}$ irinotecan on day 1 and $40-\mathrm{mg} / \mathrm{m}^{2}$ oral S- 1 twice daily on days 1-7, every 2 weeks. This dosing schedule was based on a previous phase I study [8]. Treatment continued until disease progression, intolerable toxicity, or patient's withdrawal of consent. Doses were interrupted or modified for grade 3-4 hematological toxicities and grade 2-4 nonhematological toxicities according to the protocol. Primary prophylactic granulocyte-colony stimulating factor support was not allowed. In patients who did not exhibit disease progression during completion of the 12th cycle of OIS, continuation of S-1 monotherapy was allowed at the discretion of the attending physician.

\section{Assessment}

Baseline assessment included medical history, physical examination, laboratory tests, and computed tomography (CT) of the chest, abdomen, and pelvis. Physical examination and laboratory tests were performed at each treatment cycle. For response evaluation, CT was performed every three cycles or in the presence of signs or symptoms indicating disease progression. Tumor responses were determined by local investigators according to the RECIST ver. 1.1. Toxicities were assessed every cycle and graded according to the National Cancer Institute Common Terminology Criteria for Adverse Events ver. 4.03. 


\section{Biomarker analysis}

Somatic mutations and copy number variation analyses were performed on archival tumor tissues using targeted exome sequencing. Details of methods for next generation sequencing (NGS) experiments and bioinformatics analyses are described in the Supplementary Methods. Somatic mutations were manually reviewed using Integrative Gemonic Viewers, and correlative analyses were performed between these results and clinical outcomes.

\section{Statistical analysis}

Primary endpoint was overall response rate (ORR) according to the local investigator's assessment. Secondary endpoints were progression-free survival (PFS), OS, and safety. Simon's optimal two-stage design was used to estimate sample size [10]. Estimated ORR of patients who received standard GemCis was approximately $15 \%$ according to our large retrospective analysis (P0) [11], and investigational OIS was expected to improve to $35 \%$ (P1) in ORR with two-sided $\alpha$ of 0.05 and power of $80 \%$. We assumed that $20 \%$ improvement in ORR is acceptable for further investigation of OIS in randomized phase II or III trials. After expecting a 10\% drop-out rate, target enrollment was 31 patients. In the first stage, during which 15 patients were enrolled, two patients with objective responses (complete response [CR] or PR) were required to proceed to the second stage of patient enrollment. At least 10 patients with objective responses were needed to declare OIS as effective.

PFS was defined as the time from the initiation of study treatment to disease progression or death, whichever occurred first. OS was defined as the time from the initiation of study treatment to death from any cause. The KaplanMeier method was used to estimate time-to-event variables. All efficacy parameters were analyzed based on the full analysis set, which included all patients who met the eligibility criteria. A two-sided p-value of $<0.05$ was considered statistically significant. SPSS ver. 22.0 (IBM Corp., Armonk, NY) was used to perform all statistical analyses.

\section{Ethical statement}

This multicenter, open-label, single-arm, phase II study was conducted at two tertiary referral hospitals in Korea. The study protocol was approved by the Institutional Review Board of Hallym University Sacred Heart Hospital (20151020) and Asan Medical Center (2015-1070), and all patients provided written informed consent prior to enrollment. This study was conducted in accordance with the Declaration of Helsinki and the guidelines of Good Clinical Practice (ClinicalTrial.gov identifier: NCT02527824).
Table 1. Patient characteristics

\begin{tabular}{lc} 
Characteristic & No. $(\%)(\mathrm{n}=32)$ \\
Sex & \\
Male & $24(75)$ \\
Female & $8(25)$ \\
Age, median (range, $\mathbf{y r})$ & $64(40-76)$ \\
Primary tumor site & \\
$\quad$ Intrahepatic & $13(41)$ \\
$\quad$ Gallbladder & $11(34)$ \\
$\quad$ Extrahepatic & $8(25)$ \\
Disease status & \\
Initially metastatic & $24(75)$ \\
Recurrent & $7(22)$ \\
Locally advanced unresectable & $1(3)$ \\
ECOG performance status & \\
0 & $7(22)$ \\
1 & $25(78)$ \\
Previous surgery & $8(25)$ \\
No. of metastatic sites & \\
$0-2$ & $18(56)$ \\
$3-5$ & $14(44)$ \\
Metastatic site & \\
Liver & $23(72)$ \\
Lymph nodes & $21(66)$ \\
Peritoneum & $10(31)$ \\
Lung & $7(22)$ \\
Bone & $5(16)$ \\
\hline
\end{tabular}

ECOG, Eastern Cooperative Oncology Group.

\section{Results}

\section{Patients}

Between 22 October 2015, and 16 June 2016, 32 patients with histologically documented advanced BTC were enrolled. Baseline characteristics of the patients are summarized in Table 1. Median age was 64 years (range, 40 to 76 years); 24 patients (75\%) were males. The most common condition for which study treatment was provided was initially metastatic disease $(\mathrm{n}=24,75 \%)$; this was followed by recurrent disease after curative surgery $(n=7,22 \%)$ and locally advanced unresectable disease $(n=1,3 \%)$. Among seven patients who underwent surgery, there was no patient who received adjuvant chemotherapy and adjuvant radiotherapy was given in two patients. The most common location of tumors was intrahepatic $(\mathrm{n}=13,41 \%)$, followed by gallblad$\operatorname{der}(\mathrm{n}=11,34 \%)$ and extrahepatic $(\mathrm{n}=8,25 \%)$. Liver $(\mathrm{n}=23$, $72 \%)$, lymph nodes $(n=21,66 \%)$, peritoneum $(n=10,31 \%)$, and lungs $(\mathrm{n}=7,22 \%)$ were frequent metastatic sites. At the 




Fig. 1. Waterfall plots of changes in target lesions.

time of analysis, study treatment was ongoing in seven patients without disease progression and was discontinued in 25 patients because of disease progression $(n=14)$, patient refusal $(n=4)$, adverse events $(n=4)$, and death without evidence of disease progression $(n=3)$.

\section{Efficacy}

All patients enrolled in this study had measurable disease. The waterfall plot for maximal percent changes in target lesions is presented in Fig. 1. ORR was 50\% (95\% confidence interval [CI], 34 to 66 ) according to RECIST ver. 1.1. No patient achieved CR, whereas 16 patients $(50 \%)$ achieved PR. Disease control rate, defined as the proportion of patients demonstrating CR, PR, or stable disease, was $88 \%$ (95\% CI, 71 to 96). Progressive disease was the best response in three patients $(9 \%)$. Tumor response could not be assessed in one patient $(3 \%)$ because of early death prior to the first assessment. Response rates were highest in patients with gallbladder cancer $(73 \%)$, followed by those in patients with extrahepatic $(63 \%)$ and intrahepatic cholangiocarcinoma $(23 \%$, $\mathrm{p}=0.04)$.

After a median follow-up period of 12.1 months (range, 0.3 to 16.2), median PFS was 6.8 months (95\% CI, 4.8 to 8.8 ) (Fig. 2). PFS rates at 6 and 12 months were $53 \%$ and $27 \%$, respectively. Median OS was 12.5 months ( $95 \%$ CI, 7.0 to 18.0); OS rates at 6 and 12 months were $75 \%$ and $53 \%$, respectively. Patients with extrahepatic cholangiocarcinoma had superior PFS (median, 15.3 months) compared to those with gallbladder cancer (5.9 months) and intrahepatic cholangiocarcinoma (3.0 months) (Fig. 3); this difference was margin-

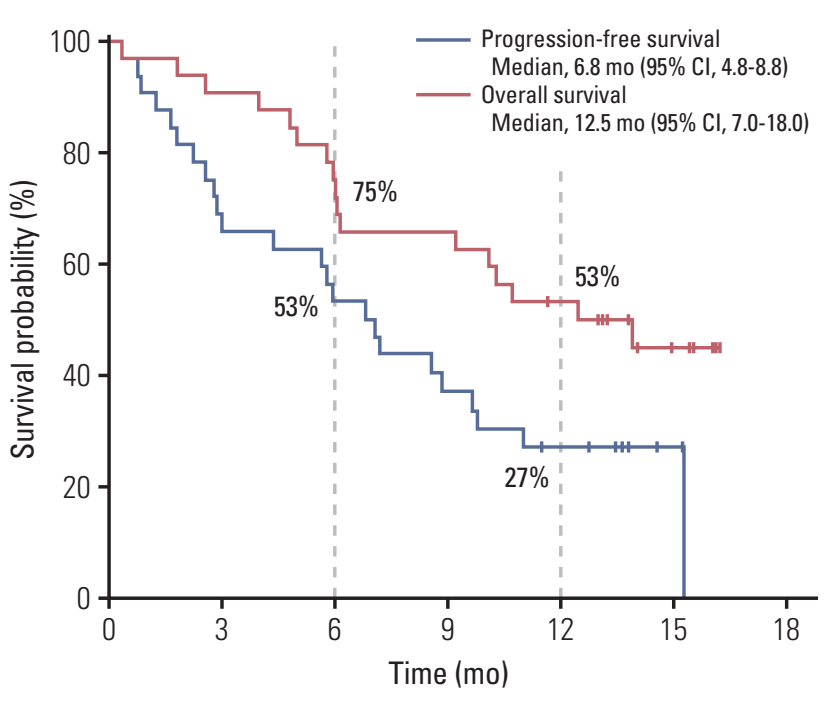

Fig. 2. Survival outcomes with oxaliplatin, irinotecan, and S-1 combination treatment. CI, confidence interval.



Fig. 3. Progression-free survival according to the primary tumor site.

ally significant $(\mathrm{p}=0.051)$. A similar trend was also observed in OS as median OS was better in patients with extrahepatic cholangiocarcinoma (median value not reached) than in those with intrahepatic cholangiocarcinoma (12.5 months) and gallbladder cancer (10.1 months); however, this was not statistically significant $(\mathrm{p}=0.21$ ).

Following progression on OIS, 50\% patients $(12 / 24)$ received second-line chemotherapy: 10 patients $(83 \%)$ received GemCis and two patients who received flouropy- 
Table 2. Adverse events occurring in $\geq 10 \%$ of the patients

\begin{tabular}{lccc} 
& \multicolumn{3}{c}{ No. $(\%)(\mathbf{n}=31)$} \\
\cline { 2 - 4 } Adverse event & Grade 1-2 & Grade 3-4 & All grades \\
Neutropenia & $17(55)$ & $10(32)$ & $27(87)$ \\
Anemia & $24(77)$ & 0 & $24(77)$ \\
Thrombocytopenia & $22(71)$ & 0 & $22(71)$ \\
Elevated AST & $11(35)$ & 0 & $11(36)$ \\
Elevated ALT & $8(26)$ & $1(3)$ & $9(29)$ \\
Hyperbilirubinemia & $5(16)$ & 0 & $5(16)$ \\
Anorexia & $16(52)$ & $1(3)$ & $17(55)$ \\
Nausea & $13(42)$ & $1(3)$ & $14(45)$ \\
Vomiting & $12(39)$ & $1(3)$ & $13(42)$ \\
Diarrhea & $16(52)$ & $2(6)$ & $18(58)$ \\
Constipation & $10(32)$ & 0 & $10(32)$ \\
Fatigue & $8(26)$ & $1(3)$ & $9(29)$ \\
Alopecia & $11(35)$ & 0 & $11(35)$ \\
Skin pigmentation & $3(10)$ & 0 & $3(10)$ \\
Peripheral neuropathy & $14(45)$ & $2(6)$ & $16(52)$ \\
Stomatitis & $6(19)$ & 0 & $6(19)$ \\
\hline Edema & $7(23)$ & 0 & $7(23)$ \\
\hline
\end{tabular}

AST, aspartate aminotransferase; ALT, alanine aminotransferase.

rimidine monotherapy (17\%). One patient underwent concurrent chemoradiation as salvage therapy.

\section{Safety profile}

Safety analysis was available in 31 patients (97\%), excluding one patient who was lost to follow-up after one cycle. A median of 12 cycles (range, 1 to 21) were administered. Study treatment was discontinued because of adverse events in four patients, and doses were interrupted and reduced in 17 $(55 \%)$ and $13(41 \%)$ patients, respectively. The mean relative dose intensity (the total delivered dose as a percentage of the targeted dose per unit time) was $87.0 \%$ (standard deviation [SD], 18.7\%) for oxaliplatin, 86.4\% (SD, 19.2\%) for irinotecan, and $86.0 \%$ (SD, 19.3\%) for S-1 during sixth cycles (S1 Fig.). Adverse events developed in $\geq 10 \%$ patients are listed in Table 2. The most common grade 3-4 adverse event was neutropenia $(n=10,32 \%)$, followed by diarrhea $(n=2,6 \%)$, and peripheral neuropathy $(n=2,6 \%)$. Despite the relatively high frequency of severe neutropenia, no patient developed febrile neutropenia.

\section{Biomarker analysis}

Eighteen of 32 patients $(56 \%)$ were available for targeted exome sequencing. TP53 ( $\mathrm{n}=7,42 \%), \operatorname{KRAS}(\mathrm{n}=5,32 \%$, including G12D in two, G13D in two, and G12A in one),
IDH1 ( $\mathrm{n}=3,16 \%)$, and ARID1A ( $\mathrm{n}=2,11 \%)$ were the most frequent genomic alterations in this study population. Although TP53 mutations were not associated with PFS ( $\mathrm{p}=0.43$ ) or OS $(\mathrm{p}=0.76), K R A S$ mutations showed a marginal relationship with worse OS ( $\mathrm{p}=0.07)$ (S2 Fig.); however, PFS was not associated with KRAS mutations ( $\mathrm{p}=0.90$ ). Although no statistical significance was observed $(\mathrm{p}=0.60)$, there was a trend toward greater tumor shrinkage $(>50 \%)$ in patients without KRAS mutations $(6 / 13,46 \%)$ compared with that in patients harboring KRAS mutations $(1 / 5,20 \%)$.

\section{Discussion}

In this single-arm, phase II study, we investigated the efficacy and safety of the triplet OIS regimen in chemotherapynaive patients with advanced BTC. Our findings suggested that OIS was well-tolerated and effective in patients with unresectable or metastatic BTC as first-line chemotherapy and that the prespecified primary endpoint was attained.

OIS demonstrated an ORR of $50 \%$, with median OS of 12.5 months, which were better than those in the pivotal phase III ABC-02 study (26\% and 11.7 months, respectively) [4]. Meanwhile, median PFS of 6.8 months with OIS in the current study seems to be inferior to 8.1 months in the ABC-02 study. 
However, this comparison should be interpreted with caution because PFS outcomes might be affected by the presence of measurable lesions or the time interval of response assessment. In contrast to the ABC- 02 study that imaging studies were conducted every 12 weeks, the current study performed response evaluation every 6 weeks. Additional support for this interpretation is provided by the Japanese phase II study as well as our previous retrospective analysis, which included response evaluation every 6 weeks and demonstrated shorter PFS (median, 5.2 to 5.8 months) despite comparable OS (10.4-11.2 months) [5,11]. Compared with these Asian studies investigating GemCis [5,11], median PFS was better with OIS (6.8 months vs. 5.2-5.8 months).

Despite the dramatic improvement in response rates with OIS compared with those achieved with GemCis in previous studies [4,5,11], differences in survival outcomes between the two regimens were not remarkable. This finding might be because of the increased rate of unfavorable clinical features in our patient population compared with that in patient population included in the ABC-02 study [4]. Only 3\% of patients in the current study had locally advanced non-metastatic disease (vs 27\% in the ABC-02 study), and all patients had measurable lesions according to the RECIST criteria (vs. $79 \%$ in the ABC-02 study). In a previous retrospective analysis involving 740 patients treated with first-line GemCis, metastatic disease and the presence of measurable lesion according to the RECIST criteria were independent poor prognostic factors [11].

OIS was generally well-tolerated, with a toxicity profile consistent with that in the previous phase I study [8]. The dose intensities of oxaliplatin, irinotecan, and S-1 were wellmaintained. Neutropenia, diarrhea, and peripheral neuropathy were the most frequent grade 3-4 toxicities associated with OIS and were manageable with dose interruption/modification and appropriate supportive care. Compared with GemCis in the ABC-02 study, OIS in our study showed increased incidence rates of grade 3-4 neutropenia (25\% vs. $32 \%$ ) and neuropathy (not reported vs. 6\%). Although increases in the rate of certain toxicities with the use of triplet combination regimens rather than doublets might be inevitable, the current study showed that the safety profile of OIS was clinically acceptable.

In the biomarker analysis using NGS, KRAS mutations were revealed as potential poor prognostic factors for patients with advanced BTC. Although the number of patients included in the biomarker analysis was too small to be conclusive, our results were consistent with those of previous studies $[12,13]$. In terms of the potential impact of the primary tumor location to the efficacy of chemotherapy in BTC, the current study showed that survival outcomes might be better in patients with extrahepatic cholangiocarcinoma than in those with gallbladder cancer or intrahepatic cholan- giocarcinoma. In previous prognostic factor analyses in patients with advanced BTC, primary tumor location was not associated with survival outcomes in patients treated with first-line GemCis [11]; however, intrahepatic cholangiocarcinoma was revealed as an independent poor prognostic factor for PFS and OS in patients treated with second-line fluorouracil-based chemotherapy [14]. These findings, together with those of the current study, suggest that the chemotherapeutic activity might differ among primary tumor sites, particularly with the use of fluorouracil-based regimens. Distinct anti-cancer activities between gemcitabine- and fluorouracilbased treatments in different primary tumor locations should therefore be investigated in future studies.

The current study had an inherent limitation as a singlearm study based on small number of patients. Considering the high heterogeneity in clinical and genetic features of patients with BTC [15], cross-study comparisons using data from single-arm studies are difficult as heterogeneity might independently impact the clinical outcomes. Another drawback of this study is that sample size calculation was based on the results of our previous retrospective analysis, not on those of the pivotal randomized trial, considering the potential ethnic discrepancies on the activity of chemotherapy. To assess the clinical relevance and future development strategy of OIS in patients with advanced BTC, therefore, a randomized trial with a control arm including GemCis is necessary.

In conclusion, triplet OIS regimen comprising oxaliplatin, irinotecan, and S-1 was feasible and effective as first-line chemotherapy in patients with unresectable or metastatic BTC. Further evaluation of OIS in randomized studies is warranted.

\section{Electronic Supplementary Material}

Supplementary materials are available at Cancer Research and Treatment website (http://www.e-crt.org).

\section{Conflicts of Interest}

S-1, irinotecan, and oxaliplatin were generously provided by Jeil Pharmaceuticals.

\section{Acknowledgments}

This study was supported in part by Hallym University Research Fund (HURF-2015-38; HURF-2016-41), Anyang, Korea and grants from the Asan Institute for Life Sciences at Asan Medical Center in Seoul, Korea (2015-0753, 2017-728). 


\section{Author Details}

${ }^{1}$ Department of Oncology, Asan Medical Center, University of Ulsan College of Medicine, Seoul, ${ }^{2}$ Department of Internal Medicine, Hallym University Sacred Heart Hospital, Hallym University College of Medicine, Anyang, ${ }^{3}$ Asan Institute for Life Science, University of
Ulsan College of Medicine, Seoul, ${ }^{4}$ Department of Pathology, Asan Medical Center, University of Ulsan College of Medicine, Seoul, Departments of ${ }^{5}$ Radiology and ${ }^{6}$ Pathology, Hallym University Sacred Heart Hospital, Hallym University College of Medicine, Anyang, Korea

\section{References}

1. Siegel RL, Miller KD, Jemal A. Cancer statistics, 2016. CA Cancer J Clin. 2016;66:7-30.

2. Jung KW, Won YJ, Oh CM, Kong HJ, Lee DH, Lee KH. Prediction of cancer incidence and mortality in Korea, 2017. Cancer Res Treat. 2017;49:306-12.

3. Edge SB, Compton CC. The American Joint Committee on Cancer: the 7th edition of the AJCC cancer staging manual and the future of TNM. Ann Surg Oncol. 2010;17:1471-4.

4. Valle J, Wasan H, Palmer DH, Cunningham D, Anthoney A, Maraveyas A, et al. Cisplatin plus gemcitabine versus gemcitabine for biliary tract cancer. N Engl J Med. 2010;362: 1273-81.

5. Okusaka T, Nakachi K, Fukutomi A, Mizuno N, Ohkawa S, Funakoshi A, et al. Gemcitabine alone or in combination with cisplatin in patients with biliary tract cancer: a comparative multicentre study in Japan. Br J Cancer. 2010;103:469-74.

6. Van Cutsem E, Moiseyenko VM, Tjulandin S, Majlis A, Constenla M, Boni C, et al. Phase III study of docetaxel and cisplatin plus fluorouracil compared with cisplatin and fluorouracil as first-line therapy for advanced gastric cancer: a report of the V325 Study Group. J Clin Oncol. 2006;24:4991-7.

7. Conroy T, Desseigne F, Ychou M, Bouche O, Guimbaud R, Becouarn $\mathrm{Y}$, et al. FOLFIRINOX versus gemcitabine for metastatic pancreatic cancer. N Engl J Med. 2011;364:1817-25.

8. Han B, Jung JY, Kim HS, Cho JW, Kim KC, Lim H, et al. A dose-finding study for oxaliplatin, irinotecan, and S-1 (OIS) in patients with metastatic or recurrent gastrointestinal cancer.
Cancer Chemother Pharmacol. 2016;78:949-58.

9. Eisenhauer EA, Therasse P, Bogaerts J, Schwartz LH, Sargent D, Ford R, et al. New response evaluation criteria in solid tumours: revised RECIST guideline (version 1.1). Eur J Cancer. 2009;45:228-47.

10. Simon R. Optimal two-stage designs for phase II clinical trials. Control Clin Trials. 1989;10:1-10.

11. Kim BJ, Hyung J, Yoo C, Kim KP, Park SJ, Lee SS, et al. Prognostic factors in patients with advanced biliary tract cancer treated with first-line gemcitabine plus cisplatin: retrospective analysis of 740 patients. Cancer Chemother Pharmacol. 2017;80:209-15.

12. Robertson S, Hyder O, Dodson R, Nayar SK, Poling J, Beierl $\mathrm{K}$, et al. The frequency of KRAS and BRAF mutations in intrahepatic cholangiocarcinomas and their correlation with clinical outcome. Hum Pathol. 2013;44:2768-73.

13. Churi CR, Shroff R, Wang Y, Rashid A, Kang HC, Weatherly $\mathrm{J}$, et al. Mutation profiling in cholangiocarcinoma: prognostic and therapeutic implications. PLoS One. 2014;9:e115383.

14. Kim BJ, Yoo C, Kim KP, Hyung J, Park SJ, Ryoo BY, et al. Efficacy of fluoropyrimidine-based chemotherapy in patients with advanced biliary tract cancer after failure of gemcitabine plus cisplatin: retrospective analysis of 321 patients. Br J Cancer. 2017;116:561-7.

15. Nakamura H, Arai Y, Totoki Y, Shirota T, Elzawahry A, Kato $\mathrm{M}$, et al. Genomic spectra of biliary tract cancer. Nat Genet. 2015;47:1003-10. 